E.S Sripta $_{\text {Romanica }}$

vol. 6 (2018): 54-64

\title{
La construction absolve dans les textes juridiques français et italiens et dans leur traduction
}

\author{
Absolute constructions in \\ French and Italian legal texts and in their translation
}

Zuzana Honová

Université d'Ostrava, République tchèque

\begin{abstract}
Résumé : Cet article traite de la construction absolve dans les textes juridiques français et italiens. II vise à montrer, sur la base d'une étude comparative, les différences de fréquence, de fonction et d'emploi observables au niveau de cette construction dans un corpus de textes législatifs, soit dans les codes de procédure pénale français et italien. En outre, il se propose de comparer les résultats obtenus avec l'analyse de la traduction du code de procédure pénale français vers l'italien et d'examiner les stratégies auxquelles le traducteur a eu recours.
\end{abstract}

Mots-clés: construction absolve, participe passé, participe présent, participe passé composé, traduction.

Abstract: The article deals with the issue of absolute constructions in French and Italian legal texts. Based on a comparative study, it aims to show the differences concerning their frequency, function and use in the corpus of legislative texts, specifically in French and Italian codes of criminal procedures. Furthermore, it proposes to compare the results with the analysis of the translation of the French code of criminal procedures into Italian and examine the strategies used by the translator.

Keywords: absolute construction, past participle, present participle, composed past participle, translation.

\section{Introduction}

Les textes juridiques se caractérisent, entre autres, par une fréquence élevée de constructions participiales, à la différence des textes rédigés dans la langue commune et ou dans d'autres langues spécialisées. Le participe y joue un rôle important et peut prendre différentes valeurs. Cet article se concentre sur l'emploi du participe (présent et passé) dans les constructions absolves, qui sont relativement fréquentes dans les textes juridiques français ainsi qu'italiens. II vise à signaler les différences entre les deux langues, que ce soit au niveau de la fréquence du participe, de son fonctionnement ou au niveau de sa position dans la phrase. De plus, il se propose d'analyser l'emploi du participe dans la construction absolve du point de vue de la traduction du français vers l'italien. 


\section{Construction absolue}

La problématique de la construction absolve a été traitée par plusieurs linguistes et nous tenons particulièrement à évoquer l'étude de Suzanne Hanon (1989), qui définit les constructions absolves comme " des constructions constituées par deux termes solidaires qui sont en rapport de sujet et de prédicat logiques ou nexus ॥ (1989: 9). De même, Riegel et al. constatent que «les constructions absolves associent deux termes dans une relation prédicative, sans expliciter par une marque formelle leur rapport entre elles, ni avec le reste de l'énoncé ॥ (2007 : 192). Une définition similaire est présentée par Grevisse : «la proposition absolve se caractérise par le fait qu'elle est constituée d'un sujet et d'un prédicat, mais sans mot introducteur et sans verbe conjugué, et qu'elle a une fonction dans la phrase ॥ (1993: 351). II convient de souligner un certain flottement terminologique : à côté de la "construction absolve " employée par Hanon, nous avons le terme de "proposition absolve " préféré par Grevisse qui ajoute que, dans certaines situations, on peut utiliser également les termes de "participe absolu» ou "proposition participe" (1993: 351). Le Goffic emploie les termes « sous-phrase participiale » ou " proposition participiale » (1994: 487-488). Le terme de "construction participiale absolve» est utilisé surtout par Eva Havu et Michel Pierrard. Ils soulignent que, d'une part, il y a un rapport de dépendance par rapport à la prédication régissante (car elle ne peut exister seule bien qu'elle constitue une prédication) et que, d'autre part, il y a une absence de marque syndétique du rapport entre la construction participiale absolve (CPA) et la proposition régissante, parce qu'aucun connecteur de type adverbial, prépositionnel ou conjonctionnel n'indique obligatoirement cette relation (2016:2).

En ce qui concerne les grammairiens italiens, ils emploient le plus souvent le terme de "participio assoluto" ". La Fauci parle du " costrutto participiale assoluto " dans lequel le prédicat est exprimé par le seul participe passé et "tra esso e la proposizione principale cui si connette, non si dà condivisione, per rinvio, di elementi argomentali " (2009: 66). De même, Simone mentionne les "strutture assolute ", constatant que "con l'espressione strutture assolute si indicano sintagmi e costrutti che non hanno rapporti di dipendenza sintattica con altri costituenti della frase e con il resto dell'enunciato in cui compaiono ॥ (Simone 2006: 2082).

II s'agit de constructions dans lesquelles le sujet est d'habitude un substantif, un syntagme nominal ou un pronom, et où le prédicat peut être un adjectif, un groupe prépositionnel ou une forme verbale non conjuguée, souvent un participe (passé ainsi que présent3). II faut préciser à cet égard qu'en italien, la situation diffère. D'une part, ce n'est que le participe passé, sauf exception, qui peut être prédicat d'une construction absolve; d'autre part, en italien, il existe aussi le gérondif absolu. Ainsi, on parle des propositions participiales et gérondivales, classées par les grammairiens italiens parmi les propositions implicites.

Ce qui distingue donc la construction absolve des autres constructions participiales est le fait que le participe absolu possède un sujet propre qui doit être

\footnotetext{
1 Cf. Dardano, Trifone : " il participio ha un suo soggetto [...], diverso dal soggetto della principale, rispetto alla quale è sintatticamente autonomo ; si chiama perciò participio assoluto ॥ (1995: 460).

2 http://www.treccani.it/enciclopedia/strutture-assolute (Enciclopedia-dell\%27ltaliano)/.

3 Cf. également Hanon : «le sujet est le plus souvent un substantif, le prédicat est de nature verbale, mais ce n'est jamais un verbe conjugué, ni un infinitif, d'où le caractère nominal de ces constructions ॥ (1989:9).
} 
différent de celui de la proposition principale. Les grammaires françaises mentionnent le plus souvent les exemples suivants:

Le chat parti, les souris dansent.

Dieu aidant, je vaincrai.

Il est évident que ces constructions sont plutôt utilisées dans le registre soutenu qui est aussi celui des textes juridiques où nous rencontrons, le plus souvent, des constructions du type :

Le jour fixé, l'affaire revient devant le juge (CPCF, art. 131-11).

en français, ou :

Chiusa la votazione, il presidente scrive e sottoscrive il dispositivo $(\mathrm{CPCl}$, art.

276).

en italien. Dans les deux exemples mentionnés ci-dessus, la construction absolve se trouve en position initiale, exprimant l'antériorité par rapport à la proposition régissante. À la différence du français où nous constatons l'ordre des mots suivant: sujet - prédicat, en italien, le participe passé précède toujours le sujet. Dans les deux langues, la construction absolve constitue une prédication secondaire, c'est-à-dire qu'il y a une relation prédicative entre les deux termes qui la composent, néanmoins elle ne peut pas fonctionner seule et se situe par conséquent dans un rapport de dépendance (subordination) par rapport à la proposition régissante (Hanon, 1989 : 59).

\section{Analyse du corpus}

Nous avons soumis à une étude comparative un corpus de textes législatifs français et italiens comprenant 400 articles du code de procédure pénale français et le même nombre d'articles du code de procédure pénale italien. Étant donné que le participe (présent etpassé) est une forme verbale fréquente dans les textes juridiques des deux langues, nous avons concentré notre attention exclusivement sur le participe employé dans les constructions absolves: sa fréquence y est moins élevée que dans d'autres types de constructions (par exemple, dans les constructions détachées), mais non négligeable.

\subsection{Le cas du français}

Ainsi, dans le corpus examiné, nous avons relevé 28 occurrences de participes employés comme prédicat d'une construction absolve, dont 15 participes passés, 6 participes passés composés et 7 participes présents. Parmi ces derniers, nous n'avons pas inclus les formules stéréotypées, typiques du langage juridique et notamment des textes législatifs, telles que le cas échéant, nonobstant, etc., qui représentent une quarantaine d'occurrences dans le corpus : dans le français contemporain, elles ont plutôt une valeur adverbiale ou prépositionnelle.

En ce qui concerne leur fonction grammaticale, Havu et Pierrard soulignent que les constructions participiales absolves peuvent soit décrire des états généralement associés à des êtres humains ou à des objets (Marie est assise, les yeux fermés), ce qui n'est pas le cas des textes juridiques, soit établir une relation de causalité (relation cause - effet) ou une relation de temporalité entre deux prédications : dans 
ce cas, il s'agit d'«interprétations d'ordre descriptif ou circonstanciel» (Havu, Pierrard, $2016: 5)$.

Concernant le participe passé, il apparaît dans les textes législatifs français dans les phrases du type :

Passé ce délai, l'huissier de justice en est déchargé (CPCF, art. 656).

L'instruction à l'audience terminée, la partie civile est entendue en sa demande [...] (CPPF, art. 460).

En position initiale, la construction absolve reflète l'enchaînement logique des événements et exprime l'antériorité par rapport au prédicat principal qui la suit (elle pourrait être précédée d'un marqueur de temps comme une fois, à peine, etc.). Dans le cas des constructions formées à partir du participe passé relevées dans notre corpus, c'est bien, dans tous les cas, la valeur temporelle qui est exprimée, c'est-àdire l'antériorité par rapport au verbe de la proposition principale, ce qu'indique d'ailleurs la position initiale de ces constructions. Nous constatons que le sujet de la construction absolve peut être antéposé ou postposé au participe passé, l'antéposition étant plus fréquente. Néanmoins, nous rencontrons également des constructions absolves en position finale, dans les structures du type :

Les experts sont choisis parmi les personnes physiques ou morales qui figurent soit sur une liste nationale établie par le bureau de la Cour de cassation, soit sur une des listes dressées par les cours d'appel, le procureur général entendu (CPPF, art. 157).

La position de la construction absolve ne semble donc pas influer sur sa valeur: en effet, qu'elle soit antéposée ou postposée, elle a une valeur temporelle. À notre avis, la dimension fonctionnelle de l'énoncé doit être prise en compte. On remarque que, la construction absolve "le procureur général entendu» constituant l'information nouvelle, rhème de l'énoncé, elle ne pourrait pas se trouver en position initiale où se situe le thème, c'est-ò-dire «les experts ». En effet, l'information connue délivrée dans l'article précédent, intitulé « De l'expertise », est de la teneur suivante :

Les experts procèdent à leur mission sous le contrôle du juge d'instruction ou du magistrat que doit désigner la juridiction ordonnant l'expertise (CPPF, art. 156).

Outre le participe passé, le participe présent peut aussi constituer le prédicat de la construction absolve dans les structures du type :

Cette décision peut être renouvelée selon la même procédure, sous réserve des dispositions de l'article 145-3, la durée totale de la détention ne pouvant excéder un an (CPPF, art. 145-1).

Les dispositions des articles 122 à 124 et 126 à 134 sont alors applicables, les attributions du juge d'instruction étant exercées par le juge de l'application des peines (CPPF, art. 722-2).

S'agissant des constructions asyndéthiques, il n'y a aucun lien explicite entre les constructions absolves et la proposition principale. La valeur de ces constructions dépend, en général, du contexte, mais aussi souvent de leur position dans la phrase. Dans les exemples cités, il ne s'agit pas de la valeur causale, typique des constructions avec un participe présent en position initiale, comme Le vélo étant un véhicule, le cycliste doit respecter le code de la route; ces constructions absolves 
en position finale expriment la simultanéité par rapport à la proposition régissante : à notre avis, elles servent à apporter une précision, une information complémentaire sur la situation désignée par le verbe de la proposition régissante (dans ce cas, sur la décision du juge des libertés et de la détention ${ }^{4}$ ).

Finalement, le participe passé composé peut également fonctionner comme prédicat de la construction absolve dans les textes juridiques français. Cela se trouve assez fréquemment dans des phrases du type :

Dans les autres cas, à titre exceptionnel, le juge des libertés et de la détention peut décider de prolonger la détention provisoire [...], l'avocat ayant été convoqué selon les dispositions du deuxième alinéa de l'article 114 (CPPF, art.145-1).

Le participe passé composé exprime toujours l'antériorité par rapport à la proposition principale. D'après Riegel, « à l'actif, il exprime l'accompli ; au passif, il exprime plutôt l'état résultant de l'achèvement du procès " (2007 : 344). II peut dénoter également une relation causale, et ce même s'il est postposé au verbe de la proposition principale, comme on le voit dans l'exemple suivant:

Ces dispositions sont aussi applicables aux personnes mentionnées à l'article 706-3 qui, victimes d'une atteinte à la personne prévue par cet article, ne peuvent à ce titre prétendre à la réparation intégrale de leur préjudice, les faits générateurs de celui-ci ayant entraîné une incapacité totale de travail inférieure à un mois (CPPF, art. 706-14).

\subsection{Le cas de l'italien}

Dans l'ensemble des textes de notre corpus issus du code de procédure pénale italien, nous avons dénombré 99 occurrences de participes passés, aucune occurrence de participe présent, un seul gérondif passé et deux occurrences de gérondifs présents en construction absolve. Même si l'on déduit de cet ensemble les constructions absolves pouvant être considérées comme des formules stéréotypées (du type salvo quanto previsto), fréquentes dans les textes de ce type, il nous reste toujours une soixantaine d'occurrences, ce qui représente tout de même une quantité importante : on constate ainsi que la fréquence du participe passé utilisé comme prédicat de la construction absolve est nettement supérieure dans les textes législatifs italiens en comparaison avec leurs pareils rédigés en français.

\begin{tabular}{|l|c|l|c|}
\hline \multicolumn{2}{|c|}{ CPPF } & \multicolumn{2}{c|}{ CPPI } \\
\hline participe passé & $\mathbf{1 5}$ & participio passato & $\mathbf{9 9}$ \\
\hline participe présent & $\mathbf{7}$ & participio presente & $\mathbf{0}$ \\
\hline participe passé composé & $\mathbf{6}$ & - & - \\
\hline \multirow{2}{*}{ gérondif } & \multirow{2}{*}{$\mathbf{0}$} & gerundio passato & $\mathbf{1}$ \\
\cline { 3 - 4 } & & gerundio presente & $\mathbf{2}$ \\
\hline
\end{tabular}

Le participe passé italien employé comme prédicat d'une construction absolve exprime toujours l'antériorité par rapport à la proposition principale. Quant à sa

\footnotetext{
${ }^{4}$ Herslund, en comparant le gérondif et le participe présent employé comme attribut, constate que «le gérondif dénote une situation autonome, concomitante à la situation désignée par le verbe principal, alors que le PP dénote toujours avec son verbe principal une seule situation » (2000: 88).
} 
position, il existe plusieurs variantes. Premièrement, la construction absolve se rencontre en position initiale :

\begin{abstract}
Avvenuta la revoca della costituzione a norma dei commi 1 e 2, il givdice penale non può conoscere delle spese e dei danni che l'intervento della parte civile ha cagionato all'imputato e al responsabile civile (CPPI, art. 82).

Concluse le formalità di conferimento dell'incarico, il perito procede immediatamente ai necessari accertamenti e risponde ai quesiti con parere raccolto nel verbale (CPPI, art. 227).
\end{abstract}

À cet égard, Mortara Garavelli précise que l'antéposition des propositions circonstancielles de temps correspond à la nécessité de respecter la succession logique et temporelle des événements (2001 : 89). Cela est évident dans le cas des exemples précédents; pourtant, il est possible de trouver la construction absolve postposée à la proposition principale, bien que ce soit la situation la moins fréquente dans le corpus examiné. Dans ce cas, malgré sa postposition, la construction absolve a une valeur temporelle, exprimant l'antériorité par rapport à la prédication principale.

\title{
... se la decisione spetta al procuratore generale presso la corte di cassazione, questi provvede sentito il procuratore nazionale antimafia e antiterrorismo (CPPI, art. 54-ter).
}

II giudice formula quindi i quesiti, sentiti il perito, i consulenti tecnici, il pubblico ministero e i difensori presenti (CPPI, art. 226).

II arrive assez souvent que le participe passé italien en construction absolve se trouve intercalé entre le sujet et le prédicat de la proposition principale (c'est-àdire : sujet - participe passé en construction absolve - prédicat) :

II procuratore generale, esaminati gli atti, determina quale ufficio del pubblico ministero deve procedere e ne dà comunicazione agli uffici interessati (CPPI, art. 54).

Dopo la liberazione, la misura può essere nuovamente disposta dal giudice, su richiesta del pubblico ministero, previo interrogatorio, allorché, valutati i risultati di questo, sussistono le condizioni indicate negli articoli [...] (CPPI, art. 302).

Quant au gérondif absolu, présent ou passé, il est plutôt rare dans les textes législatifs italiens. Sa valeur sémantique peut être causale, hypothétique ou concessive. Cependant, certains linguistes estiment qu'il est sémantiquement vague et peut donc assumer plusieurs interprétations (Čermák, Nádvorníková et alii, 2015 : 244).
A tal fine l'udienza può svolgersi anche in luogo diverso dal tribunale, avvalendosi il giudice, ove esistano, di strutture specializzate di assistenza o, in mancanza, presso l'abitazione della persona interessata all'assunzione della prova (CPPI, art. 398).
Si considerano violazioni della predetta disciplina le condotte per le quali, essendo stata esperita l'apposita procedura prevista dalla legge, risulta esclusa l'esistenza della speciale causa di giustificazione (CPPI, art. 204).

Néanmoins, il est employé bien plus souvent dans les textes juridictionnels surtout pour exprimer une relation causale par rapport au prédicat principal. C'est pourquoi 
I'on s'en sert notamment dans la motivation des jugements et des arrêts, pour expliquer les raisons d'une décision judiciaire.

[La Corte] ha ritenuto poi non dovuto l'assegno divorziale in favore della L., non avendo questa dimostrato l'inadeguatezza dei propri redditi ai fini della conservazione del tenore di vita matrimoniale $[\ldots]^{5}$.

\subsection{Traduction du code de procédure pénal français en italien}

Nous avons soumis à l'examen les codes de procédure pénale français et italien, ainsi que la traduction du code de procédure pénale français vers l'italien datant de 2002. Comme nous l'avons montré ci-dessus, dans le code de procédure pénale italien, la fréquence de la construction absolve est nettement plus élevée que dans le code français. On s'attendait donc à ce que ce soit également le cas dans la traduction du code de procédure pénale français vers l'italien, ce que n'a pas révélé l'analyse de notre corpus. Devant un tel constat, on peut légitimement se demander quelles en sont les raisons. Nous estimons que le traducteur des textes juridiques a cherché à être le plus précis possible et n'a pas osé trop s'éloigner de la structure syntaxique de la langue source.

Le tableau suivant récapitule les stratégies de traduction utilisées par le traducteur italien.

\begin{tabular}{|c|c|c|c|}
\hline \multicolumn{4}{|c|}{ Traduction de la construction absolue française vers l'italien } \\
\hline \multicolumn{2}{|c|}{ CPPF } & \multicolumn{2}{|c|}{ CPPF traduit vers l'italien } \\
\hline \multirow{2}{*}{ participe passé } & \multirow{2}{*}{15} & participe passé & 13 \\
\hline & & infinitif composé & 2 \\
\hline \multirow{4}{*}{ participe présent } & \multirow{4}{*}{7} & proposition principale & 1 \\
\hline & & proposition subordonnée & 1 \\
\hline & & syntagme prépositionnel & 2 \\
\hline & & participe présent & 3 \\
\hline \multirow{2}{*}{ participe passé composé } & \multirow{2}{*}{6} & infinitif composé & 4 \\
\hline & & syntagme prépositionnel & 2 \\
\hline
\end{tabular}

\subsubsection{Participe passé}

a) construction absolve en français > construction absolve en italien :

Nous avons relevé quinze occurrences de participes passés en français qui ont été traduits treize fois par le participe passé italien, toujours avec une valeur temporelle.

Elle peut également, dans tous les cas, le ministère public entendu, prononcer d'office la mise en liberté de la personne mise en examen (CPPF, art. 201).

Può inoltre in ogni caso, sentito il pubblico ministero, pronunciare d'ufficio la liberazione dell'imputato.

b) construction absolve en français > préposition dopo + infinito composto en italien : ${ }^{5} \mathrm{https}: / /$ www.iusexplorer.it/Dejure/Sentenze?idDocMaster=5348942\&idDataBanks=2\&idUnitaDoc=0\&nVi
gUnitaDoc=1 \&pagina=1 \& Navld=355729715\&lsCorr=False 
Cependant, la construction absolve française n'est pas toujours exprimée par la même construction en italien. Plusieurs fois, le traducteur préfère recourir à l'infinitif composé précédé de la préposition dopo qui marque nettement la valeur temporelle de cette construction.

L'ensemble des décisions de la cour fait l'objet d'un arrêt motivé, le ministère public entendu (CPPF, art. 290).

Le decisioni della corte sono oggetto di una pronuncia motivata, assunta dopo aver sentito il pubblico ministero.

Les parties ne peuvent être entendues, interrogées ou confrontées, à moins qu'elles n'y renoncent expressément, qu'en présence de leurs avocats ou ces derniers dûment appelés (CPPF, art. 114).

Le parti possono essere sentite, interrogate o sottoposte a confronto solo in presenza dei loro avvocati o dopo averli debitamente convocati, a meno che vi rinuncino espressamente.

c) syntagme prépositionnel en français > construction absolve en italien :

La formule française après avis du procureur général / ministère public est traduite en italien par la construction absolve formée à partir du participe passé sentito et du nom. II s'agit de la construction absolve la plus fréquente dans la version originale du code de procédure pénale italien.

\footnotetext{
Cependant, le premier président de la Cour d'appel peut, après avis du procureur général, ordonner [...] (CPPF, art. 236).

Tuttavia il primo presidente della Corte d'appello può, sentito il parere del procuratore generale, disporre [...].
}

Comme nous le voyons dans l'exemple précédent ainsi que dans l'exemple suivant, la position de la construction absolve en italien n'influe aucunement sur la valeur de celle-ci. Dans les deux cas, elle exprime l'antériorité par rapport à la prédication principale.

Après un délai de dix jours, il est procédé au jugement de la contumace (CPPF, art. 629).

Scaduto il termine di otto giorni si procede al giudizio in contumacia.

Nous constatons que, si l'on ne prend pas en considération des formules stéréotypées telles que fatto salvo (équivalent de la locution prépositionnelle française sous réserve de) ni les constructions absolues récurrentes (p.ex. sentito + syntagme nominal), il ne nous reste que deux occurrences de la construction absolve en italien, qui ne correspondent pas à une construction similaire en français. Ainsi, même dans les cas où le traducteur pourrait très bien recourir à une construction absolve dans la langue cible, il préfère ne pas s'éloigner de la structure syntaxique de l'original.

Lorsque les opérations d'expertise sont terminées, les experts rédigent un rapport [...] (CPPF, art. 166).

Quando le operazioni di perizia sono terminate, i periti redigono un rapporto [...].

Ce n'est que rarement, dans la traduction examinée, que le traducteur a recours à une construction absolve précédée de l'expression una volta : 
Lorsqu'une information est ouverte, elle exécute les délégations des juridictions d'instruction et défère à leurs réquisitions (CPPF, art. 14).

Una volta avviata l'attività istruttoria, la polizia giudiziaria esegue le attività ordinate dalle autorità giurisdizionali dell'istruzione e adempie alle loro richieste.

\subsubsection{Participe passé composé}

À la différence du français, où le participe passé composé peut fonctionner comme prédicat de la construction absolve, en italien, on emploie plutôt dans cette configuration, surtout dans les textes juridictionnels, le gerundio composto. Néanmoins, ce mode indéfini ne figure pas dans le corpus examiné. Pour traduire le participe passé composé français en italien, le traducteur a opté pour d'autres solutions telles que la locution prépositionnelle à valeur temporelle, comme dans l'exemple qui suit:

Dans les autres cas, à titre exceptionnel, le juge des libertés et de la détention peut décider de prolonger la détention provisoire [...], l'avocat ayant été convoqué selon les dispositions du deuxième alinéa de l'article 114 (CPPF, art. 145-1).

Negli altri casi, a titolo eccezionale, il juge des libertés et de la détention può decidere di prolungare la detenzione cautelare [...], a seguito di convocazione dell'avvocato ai sensi del secondo comma dell'articolo 114.

Une autre possibilité de traduction est le recours à l'infinitif composé, précédé de la préposition dopo qui souligne la valeur temporelle de cette construction:

Ce magistrat peut, après l'audition du prévenu, son avocat ayant été avisé et entendu en ses observations, s'il le demande, prononcer cette mesure dans les conditions et suivant les modalités prévues par les articles [...] (CPPF, art. 394).

Tale magistrato può, dopo avere avvisato e sentito il suo avvocato nelle proprie osservazioni, se il prevenuto lo richiede, pronunciare tale misura alle condizioni e secondo le modalità previste dagli articoli [...].

Ou encore le recours à une proposition subordonnée exprimant le temps :

Le procès verbal est transmis au procureur de la République, copie en ayant été remise à l'intéressé dans le cas prévu par l'alinéa suivant (CPPF, art. 78-3).

Il processo verbale è trasmesso al procuratore della Repubblica dopo che una copia è stata consegnata all'interessato nel caso previsto dal comma seguente.

\subsubsection{Participe présent}

L'emploi du participe présent dans une construction absolve dans les textes juridiques français est moins fréquent que l'emploi du participe passé. Dans le corpus examiné, nous avons relevé 7 occurrences de constructions absolves formées avec le participe présent français. Étant donné que, à la différence du participe passé, le participe présent italien ne peut pas fonctionner comme prédicat d'une construction absolve, sauf dans quelques formules stéréotypées à valeur adverbiale, telles que (ciò) nonostante et seduta stante (3 occurrences), le traducteur doit 
recourir à d'autres moyens linguistiques en italien, par exemple à un syntagme prépositionnel (préposition con + nom) ${ }^{6}$.

Tout juré [...] est condamné par la cour à une amende, laquelle est, pour la première fois, de 15 euros, la cour ayant la faculté de la réduire de moitié [...] (CPPF, art. 288).

Ogni giurato [...] è condannato dalla corte a un'ammenda, che è, per la prima volta di 15 euro, con facoltà della corte di ridurla alla metà [...].

Une autre solution traductologique est de recourir à une proposition principale divisée par un point-virgule, comme nous le voyons dans l'exemple suivant:

L'avocat de la personne mise en examen présente oralement des observations [...], lors d'une audience de cabinet dont est avisé le ministère public pour qu'il y prenne, le cas échéant, ses réquisitions, l'avocat ayant la parole le dernier (CPPF, art. 187-1).

L'avvocato dell'imputato presenta oralmente le osservazioni [...], nel corso di un'udienza in camera di consiglio della quale è avvisato il pubblico ministero affinché presenti le sue requisitorie; il difensore ha la parola per ultimo.

Finalement, dans l'exemple suivant, le traducteur a opté pour une proposition circonstancielle, probablement pour être plus précis, ce que montre la répétition du substantif detenzione.

Cette décision peut être renouvelée selon la même procédure, sous réserve des dispositions de l'article 145-3, la durée totale ne pouvant excéder un an (CPPF, art. 145-1).

Tale decisione può essere rinnovata con la medesima procedura, salve le disposizioni dell'articolo 145-3, senza che la durata totale della detenzione possa eccedere un anno.

\section{Conclusion}

Sur la base de l'analyse effectuée, nous constatons que la construction absolve est beaucoup plus utilisée dans les textes législatifs italiens que dans textes législatifs français. C'est le participe passé qui fonctionne le plus souvent comme prédicat de la construction absolve dans les deux langues, tandis que le participe présent et le participe passé composé sont moins fréquents. Le gérondif ne s'emploie pas dans ce type de construction en français ; même en italien, il est plutôt rare. En revanche, dans la traduction du code de procédure pénale français vers l'italien, nous constatons une présence nettement inférieure de la construction absolve dans le texte cible, dans lequel le traducteur préfère recourir à d'autres stratégies. Nous l'expliquons par le caractère même de la traduction juridique qui nécessite un degré de précision très élevé : le traducteur juridique cherche à parvenir au résultat le plus précis possible par rapport au texte source et n'ose pas s'écarter de la structure syntaxique d'origine.

\footnotetext{
${ }^{6}$ Nous n'avons pas pris en considération la formule stéréotypée le cas échéant, récurrente dans les textes juridiques, qui, à l'origine, était une construction absolve, mais qui, dans le français contemporain, est perçue comme un adverbe. Cette formule stéréotypée française est systématiquement traduite en italien par l'adverbe eventualmente, ce qui montre son caractère adverbial dans la langue contemporaine.
} 


\section{Bibliographie}

CORNU, G. (2006) : Linguistique juridique. Paris : Monchrestien.

CORTELAZZO, M. (1994): Lingue speciali. La dimensione verticale. Padova : Unipress.

ČERMÁK, P., NÁDVORNÍKOVÁ, O. (2015): Románské jazyky a čeština ve světle paralelních korpusü. Praha : Karolinum.

GREVISSE, M. (1993) : Le bon usage. Paris, Louvain-la-Neuve : Éditions Duculot.

HANON, S. (1989): Les constructions absolves en français moderne. Louvain-Paris: Éditions Peeters.

HAVU, E., PIERRARD, M. (2016) : Constructions participiales absolves et liaisons de prédications : au-delà du lien syndétique, disponible sur le site : https://www.shs-

conferences.org/articles/shsconf/pdf/2016/05/shsconf_cmlf2016_14005.pdf.

HERSLUND, M. (2000) : "Le participe présent comme co-verbe ", [in :] Langue française, $n^{\circ} 127$, p. 86-94, disponible sur le site :

http://www.persee.fr/web/revues/home/prescript/article/lfr_00238368_2000_num_127_1_1000, consulté le 2.4.2018.

LA FAUCl, N. (2009): Compendio di sintassi italiana. Bologna: II Mulino.

LE GOFFIC, P. (1994) : Grammaire de la phrase française. Paris : Hachette.

MAFFEI, S. (2002): II codice di procedura penale francese. Testo originale e traduzione in italiano, La Tribuna.

MORTARA GARAVELLI, B. (2001): Le parole e la giustizia. Divagazioni grammaticali e retoriche su testi giuridici italiani. Torino : Einaudi.

RIEGEL, M., PELLAT, J.-Ch., RIOUL, René (2006) : Grammaire méthodique du français. Paris : Presses Universitaires de France.

SIMONE, R. (2006): Fondamenti di linguistica. Roma - Bari : Laterza (1a ed. 1990), disponible sur le site : http://www.treccani.it/enciclopedia/struttureassolute_(Enciclopedia-dell\%27ltaliano)/, consulté le 2. 4. 2018.

ZINGARELLI, N. (1997): Vocabolario della lingua italiana. Bologna: Zanichelli editore.

\section{Sitographie}

http://altalex.it/

https://www.iusexplorer.it/

http://legifrance.fr/

\section{Liste des abréviations}

CPPF - Code de procédure pénale français

CPPI - Codice di procedura penale italiano

CPCF - Code de procédure civile français

$\mathrm{CPCl}$ - Codice di procedura civile italiano 